\title{
Extragalactic Supernovae and the Starformation Rate
}

\author{
Alan Pedlar \\ Jodrell Bank Observatory, University of Manchester, $\mathrm{Nr}$, Macclesfield, \\ UK, SK10 5NE
}

\begin{abstract}
.
The advantages of studying samples of supernova remnants in external galaxies will be discussed. A summary of how the remnants can be used to infer starformation rates in starburst galaxies will be given, as will the use of these remnants to probe the interstellar medium of these galaxies on parsec scales. Widefield EVN and Global VLBI measurements of remnants in the nearby starburst in M82 will be described in detail, and recent expansion velocity measurements described. The similarity between the strongest compact source in M82 and the objects recently discovered in Arp220 will be noted. The sensitivity limitations of this work will be discussed and the prospects for studies of more distant objects using Square Kilometer Array considered.
\end{abstract}

\section{Introduction}

Compared with supernova remnants studies in our own galaxy, extragalactic studies have been limited in sensitivity and linear resolution. However with the advent of VLBI, linear resolutions of order $0.05 \mathrm{pc}$ are now attainable in nearby galaxies. Despite the formidable technical difficulties, extragalactic studies of supernova remnants have a number of advantages over Galactic studies. The difficulties of establishing statistically complete samples of Galactic remnants are well known (eg Green 1984). Not only are the distances of many galactic remnants uncertain, but they have often been observed with different instruments with varying angular resolution and sensitivity. Extragalactic studies have the immediate advantage that the difference in distances to remnants with a galaxy is negligible compared to the distance of the galaxy from the observer. Hence the relative distances of a sample of remnants in a external galaxy are often the same to $<1 \%$. Furthermore as the sample in an external galaxy will usually have been observed with a single instrument, this means that the remnants have been observed with identical instrumental sensitivity and angular resolution. As the remnants are all at essentially the same distance, the latter also implies they are observed with the same linear resolution.

The first extragalactic study of remnants was carried out in the Large Magellanic cloud ( Mills et al 1984). Large samples of supernova remnants have also been studied in M31 (Braun \& Walterbos 1993) and M33 (Duric et al 1993). Most of the remnants in these galaxies have sizes of typically 10-100pc and are of relatively low brightness, corresponding to old, evolved remnants consistent with 
normal starformation rates. By considering the completeness of their sample of remnants in M31, Braun \& Walterbos estimated a supernova rate (due to stars more massive than $7 M_{o}$ ) of 1 every 80 years. In principle, because of their proximity, these remnants could be studied with very high linear resolution obtained via sparsely filled radio arrays. However the rapid decrease in brightness sensitivity with increasing angular resolution means that, with current sensitivities, few, if any, of these remnants are available for VLBI or even MERLIN studies.

To investigate samples of younger remnants it is necessary to observe galaxies with high star formation rates. When the rate of starformation is so high that it cannot be maintained in equilibrium over the lifetime of a galaxy the phenomenon is known as a 'starburst'. These galaxies contain large numbers of young remnants which are both compact $(<10 \mathrm{pc})$ and are of high radio brightness. Such populations of compact sources have been studied in a number of starburst galaxies including M82 (Unger et al 1984; Kronberg et al 1985) NGC253 (Ulvestad \& Antonucci 1997), NGC4736 (Duric \& Dittmar 1988), NGC3448 (Noreau \& Kronberg 1987), NGC2146 (Tarchi et al 2000) \& NGC4038/9 (Neff $\&$ Ulvestad 2000). In this review we discuss the possibility of using these measurements to determine supernova rates and hence, via the initial mass function (IMF), starformation rates.

\section{Starformation rates in starburst galaxies}

The most widely used diagnostic of the starformation rate is the far infra-red luminosity (eg Reike et al 1980). This is believed to be due to emission from dust grains which have been heated by photons from young stars created in the starburst. If it is assumed that most of the energy in photons emitted by young stars eventually results in heating the dust - which re-radiates it as black-body thermal continuum in photons, then the starformation rate (SFR) for star more massive than $5 M_{o}$ can be estimated (eg Cram et al 1998) from the far-infrared luminosity $\left(L_{60 \mu}\right)$ as

$$
\operatorname{SFR}\left(M \geq 5 M_{o}\right)=\frac{L_{60 \mu}}{5.1 \times 10^{23}} M_{o} y r^{-1}
$$

The starformation rate can also, in principle, be measured by observations of the properties of the UV photons from O and B stars although this method is severely compromised in many starbursts because of the extinction intrinsic to the starburst. An indirect measure of the UV photons can be made by determining the parameters of the HII regions ionised by them. This can be carried out via optical (eg $\mathrm{H} \alpha$ ) emission lines (eg Kennicutt 1983) although quantitative measurements of individual starbursts are again compromised by dust extinction.

Thermal free-free emission at $\mathrm{cm}$ wavelengths offers an alternative method of deriving the HII region parameters unaffected by dust extinction. Hence if the thermal free-free luminosity $L_{T}$ of a starburst is known, the flux of Lyman continuum photons $\sec ^{-1}\left(N_{U V}\right)$ can be estimated from (eg Condon 1992 )

$$
N_{U V} \geq 6.3 \times 10^{52} T_{e}^{-0.45} \nu^{0.1} L_{T}
$$


where $T_{e}$ is the electron temperature in units of $10^{4} \mathrm{~K}, \nu$ is the frequency in $\mathrm{GHz}$ and $L_{T}$ is the thermal free-free luminosity in units of $10^{20}$ Watt $\mathrm{Hz}^{-1} . N_{U V}$ can then be converted into a starformation rate via the relation

$$
\operatorname{SFR}\left(M \geq 5 M_{o}\right)=\frac{N_{U V}}{3.5 \times 10^{53}} M_{o} y r^{-1}
$$

The main difficulty with this method is the problem of separating the the free-free emission from the non-thermal synchrotron emission which will dominate the radio luminosity particularly at the longer wavelengths.

The diffuse non-thermal radio continuum can also be used to infer the starformation rate in galaxies. This is mainly on the basis of the surprisingly good correlation between the far-infrared and radio luminosity (eg Helou et al 1985). As the most likely source of relativistic electrons responsible for the diffuse nonthermal emission is supernova explosions, it is generally assumed that the nonthermal radio luminosity is determined by the supernova rate in a galaxy and hence the starformation rate. Thus if a Miller-Scalo IMF is assumed then a supernova rate of $\nu_{s n}$ per year will imply a starformation rate of

$$
S F R\left(M \geq 5 M_{o}\right)=25 \nu_{s n} \mathrm{M}_{\mathrm{o}} \mathrm{yr}^{-1}
$$

Hence this accounts for the tight correlation between the far infrared \& radio continuum luminosity as both measurements are linked via a common starformation rate (eg Cram et al 1998). Note that, as pointed out by Condon (1992), the starformation rate inferred from supernova rates and UV photon fluxes are sensitive to the IMF in different ways. Hence, in principle, accurate measurements of starformation rate using these methods could be used to, at least, constrain the IMF in starburst galaxies.

\section{Supernova remnants in starburst galaxies}

In the early 1980s MERLIN (Unger et al 1984) and the VLA (Kronberg et al 1985 ) discovered $\sim 50$ compact sources in the nearby starburst Messier 82 (see Figure 1). Subsequently most of these sources proved to be supernova remnants (Muxlow et al 1994) on account of their steep spectrum, parsec-scale sizes and high brightness temperatures. The benefits of studying supernova remnants in starburst galaxies can roughly divided into two areas.

Firstly those areas in which the supernova remnants give information on the starburst. As well as determining the global supernova, and hence starformation, rate, the distribution of the SNR, unaffected by extinction, shows the structure of the starburst approximately $10^{7}$ years ago. This can be compared with the HII region distribution which traces the current starburst. In addition, via absorption studies, the continuum emission from the remnants can be used as a high angular resolution probe of the ionised (Wills et al 1997), atomic hydrogen (Wills et al 1998, 2000) and molecular gas (eg $\mathrm{OH}, \mathrm{H}_{2} \mathrm{CO}$ etc) associated with the starburst.

Secondly the study of these compact sources in starburst galaxies provides unique information on the properties and evolution of supernova remnants. As noted in the introduction the sample of remnants in a given starburst are essentially at the same distance and have been observed with identical angular/linear 


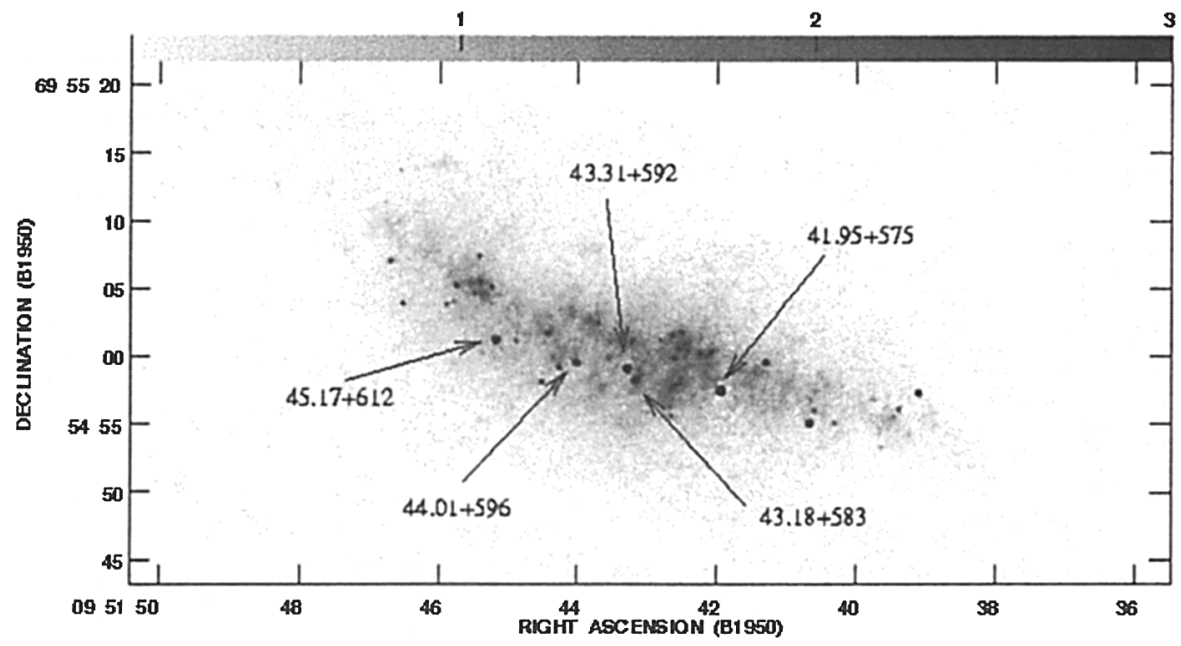

Figure 1. The $20 \mathrm{~cm}$ VLA+MERLIN radio image showing the compact radio sources in M82. The sources which have been studied with VLBI are labelled

resolution and sensitivity (For example as the starburst in M82 has a size of $<1 \mathrm{kpc}$, then assuming a distance of $3200 \mathrm{kpc}$, the differences in relative distance for the sample of remnants is less than $0.03 \%$ !). Furthermore, many of the supernova remnants in starbursts cover a unique age range. The youngest accessible supernova remnant in our galaxy appears to be Cass A with an age $\geq 300$ years. VLBI studies of extragalactic radio supernovae from different galaxies have given considerable information on the early evolution of supernova remnants. However because such observations have only been feasible with VLBI since the late 1970 s, little is known currently about the behavior of such objects with ages greater than 20 years. As we shall discuss below it seems likely that the remnants in M82 have ages ranging from 30 to 1000 years and hence observations of remnants in starbursts enables their properties to be parameterised over this age range.

\subsection{M82 - a case study}

However, as yet, most of these other starburst galaxies have not been observed with sufficiently high linear resolution to measure the sizes of supernova remnants, and often there is some contamination by HII regions which have to be eliminated either by measuring their spectrum and/ or brightness temperature. The nearest starburst galaxies are NGC253 and M82 of which the latter is by far the best studied. At present M82 is the only starburst in which both the sizes and luminosities of a large number of remnants have been measured (Muxlow et al 1994). In addition an extensive flux monitoring campaign has been carried out by Kronberg et al (2000) to investigate their radio variability. In Figure 2 


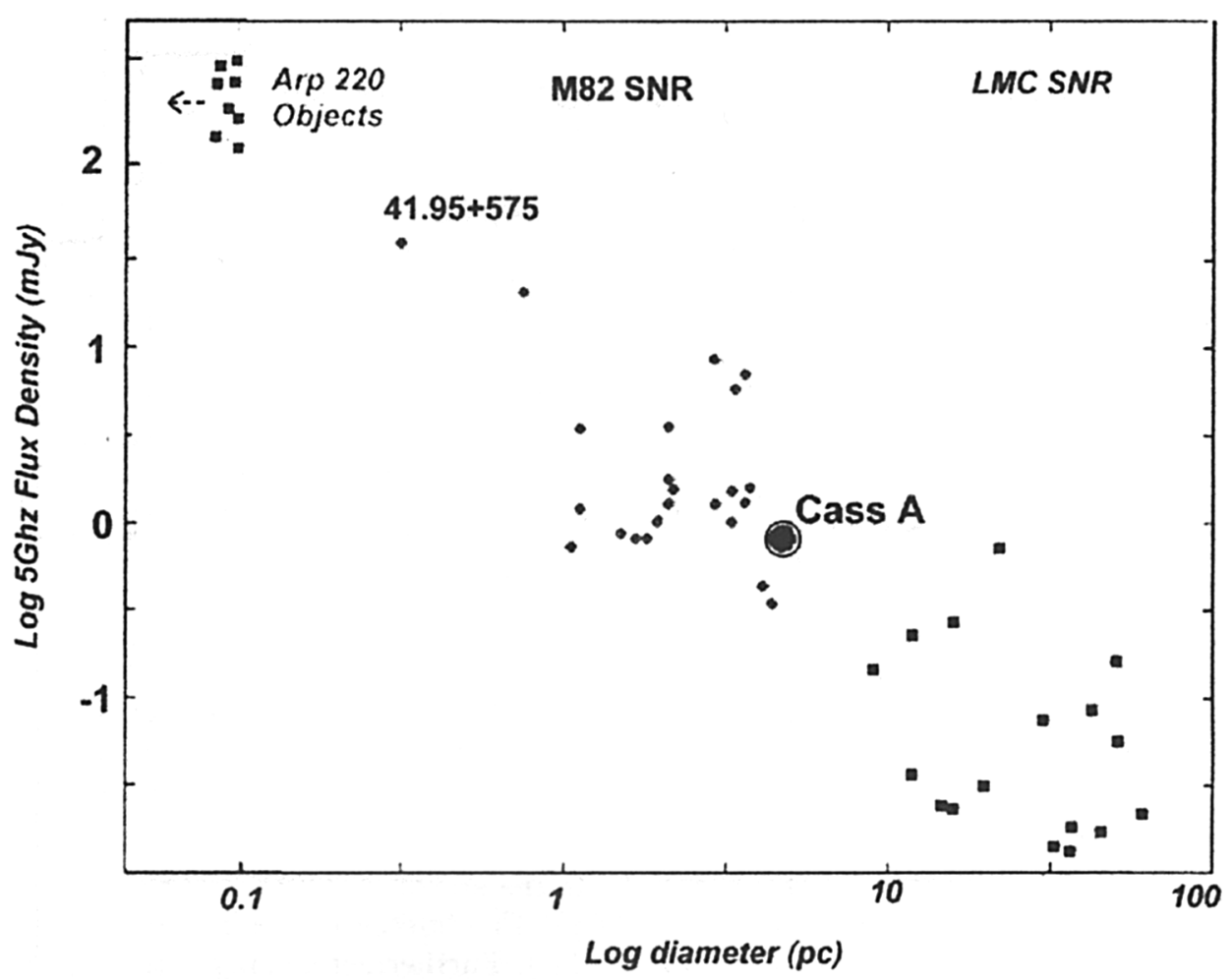

Figure 2. A plot of size vs $5 \mathrm{GHz}$ flux density for the compact sources seen in M82 compared with Cass A (from Muxlow et al 1994). The flux densities for supernova remnants in the Large Magellanic Cloud (Mills et al 1984) scaled to be at the distance of M82 are shown, together with the approximate parameters of the Arp220 estimated from Smith et al 1998.

we show a plot of the flux density vs size of the remnants in M82 compared with samples in the LMC and Arp220 (both scaled to $3.2 \mathrm{Mpc}$ ). From this plot it can be seen that most of the M82 remnants are more luminous and more compact than Cass A. One question which needed to be addressed is whether the size of the remnants is a function of age, or whether the sizes are determined by the ambient density.

One method of distinguishing between these possibilities is to measure the expansion velocities of the remnants directly. To this end EVN measurements were carried out at two epochs and an expansion velocity of one of the brighter remnants measured to be $\sim 10000 \mathrm{~km} \mathrm{~s}^{-1}$ (Pedlar et al 1999). If the remnant is in free expansion this implies an age of $\sim 35$ years, or shorter if significant deceleration has occurred. Recent Global VLBI measurements (McDonald et al 2000 ) of these remnants (Figure 3a) have angular resolutions of a few milliarcsec- 

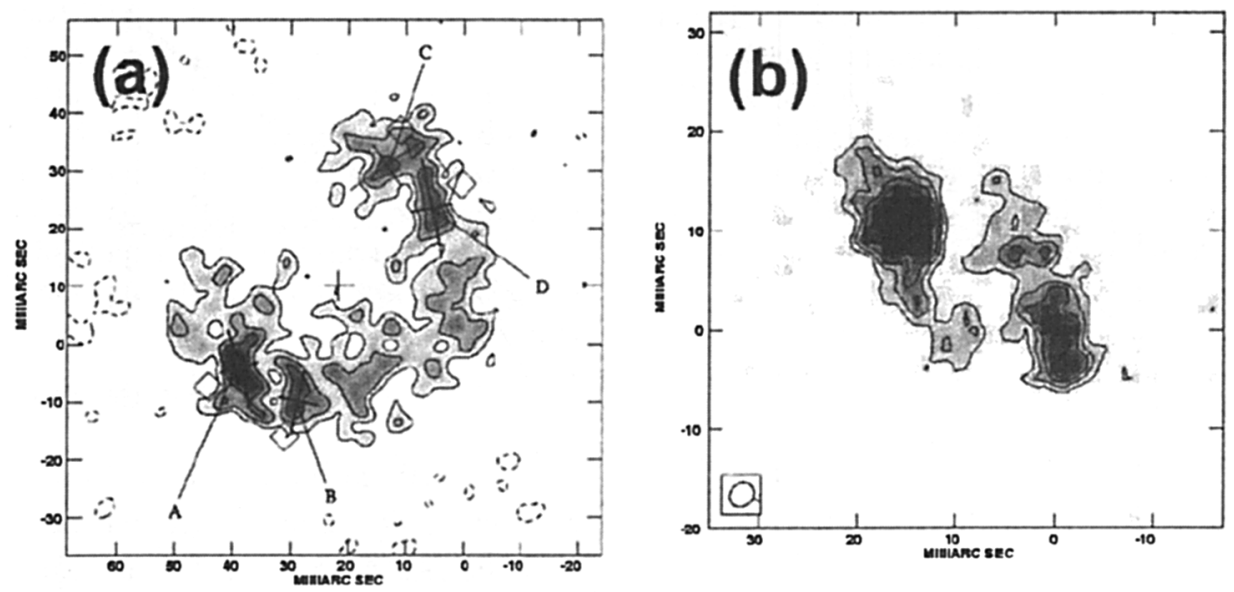

Figure 3. (a) A $20 \mathrm{~cm}$ Global VLBI image of the remnant $43.31+592$ in M82 with an angular resolution of $4 \mathrm{mas}(=0.06 \mathrm{pc})$. The positions of the 4 most prominent knots are marked with crosses, and the open diamonds indicate the position of the knots in 10 years after 1998.9 ( assuming the remnant continues to expand at $10000 \mathrm{~km} \mathrm{~s}^{-1}$ ) (b) $20 \mathrm{~cm}$ global VLBI image of $41.95+575$ - the most luminous compact source in M82. The angular resolution is $2.7 \times 2.3$ mas. (from McDonald et al 2000)

onds corresponding to $\sim 0.06 \mathrm{pc}$ and hence direct measurement of deceleration should be possible in a few years. Given that $43.31+592$ must have originated before it appeared on the early images in 1972 (Kronberg \& Wilkinson 1975) it is already possible to rule out Sedov expansion suggesting that at least this remnant is still expanding close to free expansion (Figure 4). It therefore appears that the size of at least some of the remnants give an estimate of their age, and hence assuming a common expansion velocity of $10000 \mathrm{~km} \mathrm{~s}^{-1}$, or simply assuming all the remnants more compact than Cass A are less than 330 years old, gives a supernova rate of $\sim 0.07 \mathrm{yr}^{-1}$. This can be used to calculate a starformation rate $\left(\geq 5 M_{o}\right)$ of $\sim 1.8 M_{o} \mathrm{yr}^{-1}$. This is consistent with the $\left(\geq 5 M_{o}\right)$ starformation rates derived from the FIR emission, non-thermal radio continuum and the thermal continuum all of which give values close to $2 M_{o} \mathrm{yr}^{-1}$. The supernova remnant technique will improve as the properties of the remnants are better understood. In addition, for regions with high starformation rates, it should be possible to measure the supernova rate directly by radio monitoring on timescales of a few years. Somewhat disappointingly, although M82 has now been monitored for almost two decades (Kronberg et al 2000), no new supernova has been detected. However as the expected rate, on average, is only one every $\sim 14$ years, this is not unlikely. 


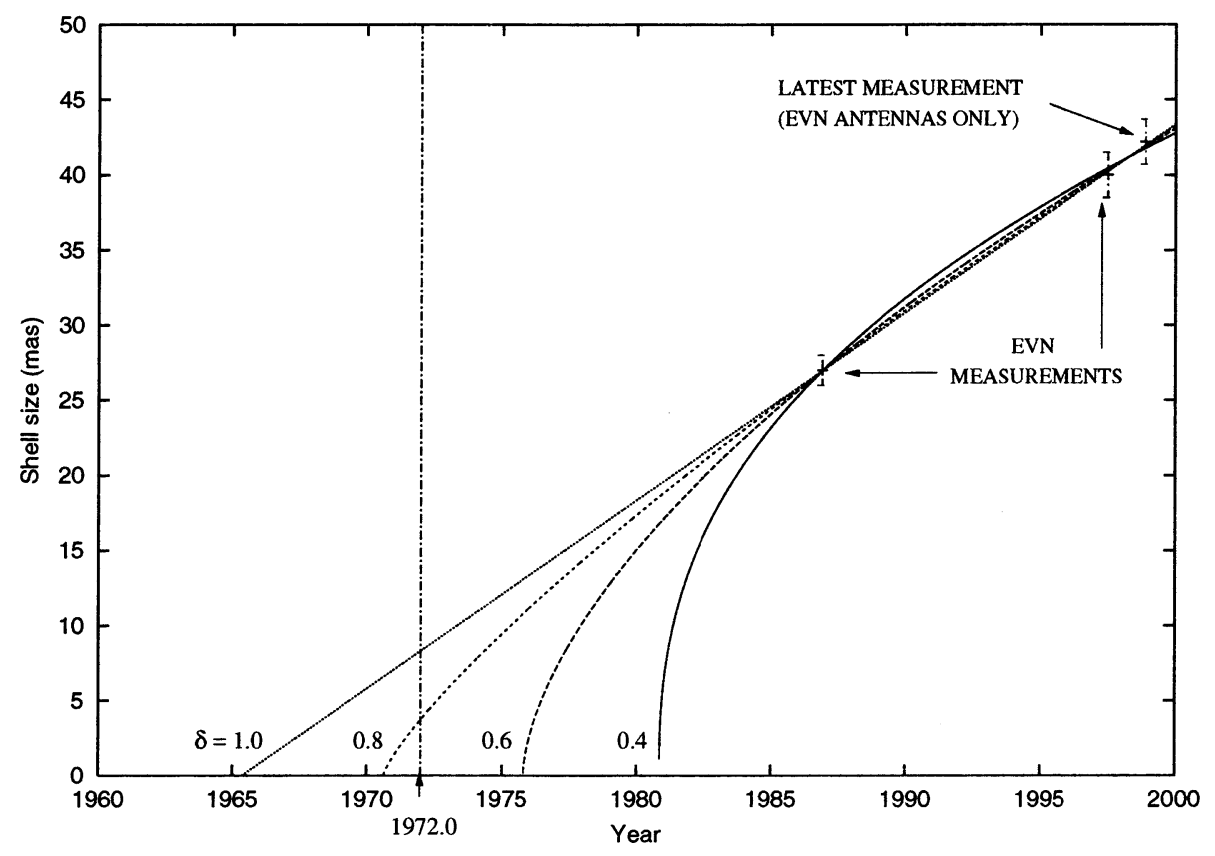

Figure 4. Size vs time plots for the compact supernova remnant 43.31+592 in M82 from McDonald et al (2000). The curves show four possible models with deceleration parameters from 0.4 to 1.0 (FreeExpansion). Note that $43.31+592$ was present in radio images taken in 1972 , which puts a lower limit on its age and rules out deceleration parameters $<0.7$.

\subsection{Compact ultra-luminous objects}

The most luminous compact source in M82 (41.9+575) appears to be anomalous when compared to the other sources. Not only is it more than an order of magnitude more luminous than typical remnants in M82, but its luminosity has been decreasing rapidly ( $8.5 \%$ per annum) since its discovery in the mid 1960s. Furthermore recent global VLBI measurements (Figure 3b) show an elongated structure which could be consistent with collimated ejection rather than a shell. Unlike the shell supernova discussed above the object is not expanding at $\sim 10000 \mathrm{~km} \mathrm{~s}^{-1}$ and limits of $<2500 \mathrm{~km} \mathrm{~s}^{-1}$ have been determined. One explanation for this object is that it is a supernova remnant confined by a high density medium, although other possibilities require investigation.

As can be seen in Figure 2, the current radio luminosity and size of $41.9+575$ appears to be intermediate between the typical remnants in M82, and the compact objects recently discovered in Arp220 (Smith et al 1998) and Mkn273 (Carilli \& Taylor 2000). Note that in the 1960 s $41.95+575$ was of comparable luminosity to the Arp220/Mkn273 objects. Thus it is possible that the compact objects seen in Arp220 and Mkn273 may be similar to $41.9+575$. 


\subsection{Prospects}

As young supernova remnants have sizes of order a few parsec, in principle milliarcsecond VLBI studies have the angular resolution to investigate remnants in starbursts up to $\sim 100 \mathrm{Mpc}$. Unfortunately the brightness temperature sensitivities of current instruments limit VLBI studies of normal remnants to a few nearby objects. Even in M82 only 10\% of the remnants can be studied. If these studies are to extend to more distant objects, an increase in sensitivity of 1 to 2 orders of magnitude is necessary. This will be provided partly by increasing the observing bandwidth of instruments such as MERLIN and the EVN, although in the longer term a large increase in the collecting area for VLBI, using technology anticipated for SKA, will also be required.

\section{References}

Braun, R. \& Walterbos, R.A.M. 1993 A\&AS, 98327

Carilli, C.L., \& Taylor, G.B. 2000 ApJ, 532, L95

Condon, J.J. 1992 ARA\&A, 30, 575

Cram, L.E. et al 1998 ApJ, 507, 155

Duric, N. et al 1993 A\&AS, 99, 217

Duric, N. \& Dittmar, N.R. 1988 ApJ, 332, L67.

Green, D.A. 1984 MNRAS, 209, 449

Helou, G. et al 1985 ApJ, 298, 7

Kennicutt, R.C. 1983 ApJ, 27254

Kronberg, P.P. \& Wilkinson, P.N. 1975 ApJ, 200, 430

Kronberg, P.P., Biermann, P., \& Schab, F.J. 1985 ApJ, 291, 693

Kronberg, P.P. et al 2000 ApJ, 535, 706

Muxlow, T.W.B. et al 1994 MNRAS, 266, 455

McDonald, A. et al 2000 MNRAS (in press)

Mills, B.Y. et al 1984 Aust.J.Phys., 37, 321

Neff, S.G. \& Ulvestad, J.S. 2000 AJ, 120, 670

Noreau, L., \& Kronberg, P.P. 1987 AJ, 93, L1045

Pedlar, A. et al 1999 MNRAS, 307, 761

Rieke, G.H. et al 1980 AJ, 238, 24

Smith, H. et al 1998 ApJ, 493,L17

Tarchi, A. et al 2000 A\&A, 358, 95

Unger, S.W. et al 1984 MNRAS, 211, 783

Ulvestad, J.S.\& Antonucci, R.J. 1997 ApJ, 488, 621

Wills, K.A. et al 1997 MNRAS, 291, 517

Wills, K.A., Pedlar, A., \& Muxlow,T.W.B. 1998 MNRAS, 298, 347

Wills, K.A. et al 2000 MNRAS, 316, 33 